\title{
Primary results of abdominal aortic aneurysm screening in the at-risk residents in middle China
}

\author{
Kun Li, Kewei Zhang, Tianxiao Li and Shuiting Zhai id
}

\begin{abstract}
Background: There is a lack of information on the epidemiological data and risk factors associated with abdominal aortic aneurysm (AAA) in Chinese population. We reported the primary results from screening five-community population in Middle China for AAA.

Methods: From March 2014 to October 2015, an AAA screening program was performed in three urban and two rural communities. These communities were randomly selected. All at-risk residents (a total of 6925) aged 40 years or older were invited to attend an ultrasound scan for AAA. At-risk population was defined as having a family history of first-degree relative diagnosed with AAA; or smoking and aged 55 years or older; or having a history of CAD, cerebrovascular disease, hypercholesterolemia, obesity $\left(B M I \geqq 26 \mathrm{~kg} / \mathrm{m}^{2}\right.$ ) or hypertension.

Results: The study investigated 5402 subjects and the mean age of them was $61.2 \pm 10.4$ years old. It included 2847 women aged $62.5 \pm 10.4$ years and 2555 men aged $59.7 \pm 10.2$ years. The mean maximum infrarenal aortic diameter (Max-IAD) was $15.0 \pm 2.7 \mathrm{~mm}$ (from 4.1 to $51.5 \mathrm{~mm}$ ). Eighteen people (aged $68.0 \pm 10.4$ years) with AAAs were detected (prevalence rate was $0.33 \%)$, and the prevalence rate in males was higher than in females (0.55\% vs $0.14 \%$, respectively, $P=0.009$ ). Additionally, the screened subjects aged 55 to 75 years had a higher prevalence rate of AAA than other age groups $(0.51 \%$ vs $0.11 \%$, respectively, $P=0.016)$.

Conclusion: The mean Max-IAD of the screened population in Middle China was apparently small by comparison with other reports. The result of low prevalence rate of AAA didn't support routine screening in Chinese population. The at-risk males aged 55 to 75 years should be targeted for further screening.
\end{abstract}

Keywords: AAA, Max-IAD, Prevalence rate, Ultrasound screening

\section{Background}

Abdominal aortic aneurysm (AAA) is usually undiagnosed due to no symptoms before rupture. Its mortality rate is high even if the operation time is allowed [1-3]. A population-based ultrasonography screening program has been advocated because screening for AAA has been shown to reduce AAA-related mortality and provide an opportunity to modify cardiovascular risk and improve the general health of the screening population [4]. Several randomized controlled trials suggested that early detection of AAA by ultrasound screening could significantly reduce

\footnotetext{
*Correspondence: Zhaishuiting2008@163.com

Department of Vascular and Endovascular Surgery, Henan Provincial People's Hospital, Fuwai Central China Cardiovascular Hospital, NO.7 Weiwu Road, Zhengzhou 450003, Henan, People's Republic of China
}

all-cause mortality in men aged $\geq 65$ years and the cost of treatment for AAA during long-term follow-up [5-8].

Considering the balance between cost-effectiveness and incidence, high-risk population shall be targeted for screening trial. It has been proved that the increased risk of AAA includes advanced age, a history of smoking, atherosclerotic burden, and a family history of aneurysmal disease [7, 9]. Several reports demonstrated that Asians had a lower prevalence rate of AAA than that was reported in Caucasians after adjusting for all other known influencing factors [10-14]. However, there is a lack of information on the epidemiological data and risk factors of AAA disease in Chinese mainland. Consequently, a community-based screening program for AAA was performed in residents of randomly selected urban and rural 
communities in Middle China because of the highest uptake. The aim of this study was to report the primary results of the screened population for the prevalence rate of AAA and associated risk factors with AAA in Chinese population.

\section{Methods}

From March 2014 to October 2015, an AAA screening program was performed in five communities. Five communities (including three urban and two rural communities) were randomly selected from Zhengzhou city in Middle China. The geographic areas of our screening program contain a significant difference between urban and rural areas because of the different socioeconomic status, the dietary and lifestyle habits. The total population aged 40 years or older was estimated at 12,550 based on demographic statistics. A population-based sample of subjects aged 40 years or older was enrolled and invited to complete a risk-factor questionnaire in screening sites or by telephone before screening.

The at-risk population was enrolled according to the assessment of risk-factor questionnaires which included demographics, residential area, weight, height, self-reported smoking, self-reported drinking history (alcohol consumption, contained years of drinking), hypertension or diabetes (as a history of this disease treated through diet or medication), hyperlipidemia, coronary artery disease (CAD, as a history of myocardial infarction), angina pectoris of coronary revascularization, cerebrovascular disease (as a history of previous transient ischemic attack or stroke), and family history (first-degree relative diagnosed with AAA). The weight and height were used to calculate body mass index (BMI). Self-reported smoking was defined as a lifetime used 100 cigarettes and contained information of daily cigarette consumption (packs/day) in addition to years of smoking. Twelve thousand and one hundred questionnaires were collected for further risk assessment. At-risk population was defined as having a family history of first-degree relative diagnosed with AAA, smoking population aged 55 years or older, or having a history of CAD, cerebrovascular disease, hypercholesterolemia, obesity (BMI $\geqq$ $26 \mathrm{~kg} / \mathrm{m}^{2}$ ) or hypertension. The final cohort included 5402 screened subjects $(78 \%)$ in the 6925 at-risk eligible subjects.

Before ultrasound scan, all screened individuals had received a physical examination including blood pressure, weight and height. The systolic blood pressure and diastolic blood pressure were measured in both upper extremities. When blood pressure in both upper extremities measurements inconsistent, the higher one was used. The blood test was carried out including fasting blood-glucose, triglyceride, serum total cholesterol (TC), low density lipoproteins (LDL), high density lipoprotein (HDL), and homocysteine level.
All screened individuals underwent an ultrasound screening of AAA to identify the maximum anteroposterior diameter of the infrarenal aorta according to the leading edge principle in longitudinal section of aorta [15]. Ultrasonic examination was performed by an ultrasound machine (SonoSite 180 PLUS, USA) with a 3.5 $\mathrm{MHz}$ transducer. AAA was identified as a maximum infrarenal aortic diameter (Max-IAD) exceeded $30 \mathrm{~mm}$ or had $50 \%$ increase or more in diameter compared with the mean normal. The data was entered into a specific database.

\section{Statistical analysis}

SPSS 19.0 software package was used for the statistical analyses in this study. Student $t$ test or one-way analysis of variance (ANOVA) was used to compare quantitative variables. The difference was statistically significant $(P<0.05)$.

\section{Results}

\section{Characteristics of the enrolled population}

The final at-risk cohort contained 5402 screened subjects aged $61.2 \pm 10.4$, including 1682 subjects (31.13\%) under 55 years old, 3166 subjects $(58.61 \%)$ aged 55 to 75 , and 554 subjects $(10.26 \%)$ over 75 years old. As shown in Table 1, the urban residents accounted for $48.17 \%$ ( $n=$ 2602). Moreover, 1481 subjects $(27.42 \%)$ had a history of stroke, 983 subjects had CAD (18.20\%), 1681 subjects (31.12\%) had a history of diabetes, 3685 subjects $(68.22 \%)$ had hypertension, 1275 subjects $(23.60 \%)$ had self-reported smoking, and 1142 subjects (21.14\%) had drinking history in total individuals. The smoking rate for men was higher than that for women $(P<0.001$, Table 1$)$, and approximately

Table 1 Characteristics of screened at-risk population by gender

\begin{tabular}{lllll}
\hline Characteristics & Overall & Male & Female & $P$ \\
\hline $\begin{array}{l}\text { Screened } \\
\text { individuals, } \%\end{array}$ & $5402(100)$ & $2555(47.30)$ & $2847(52.70)$ & 0.615 \\
Age & & & & $0.009^{*}$ \\
Overall age & $61.2 \pm 10.4$ & $59.7 \pm 10.2$ & $62.5 \pm 10.4$ & \\
Age without AAA & $61.2 \pm 10.4$ & $59.6 \pm 10.2$ & $62.5 \pm 10.3$ & \\
Age with AAA & $68.0 \pm 10.4$ & $57.1 \pm 10.5$ & $71.3 \pm 11.2$ & \\
Screening areas & & & & $0.041^{*}$ \\
Urban,\% & $2602(48.17)$ & $1216(22.51)$ & $1386(25.66)$ & \\
Rural,\% & $2800(51.83)$ & $1339(24.79)$ & $1461(27.05)$ & \\
History of stroke,\% & $1481(27.42)$ & $690(12.77)$ & $791(14.64)$ & 0.063 \\
CAD, \% ${ }^{\text {a }}$ & $983(18.20)$ & $368(6.81)$ & $615(11.38)$ & $0.004^{*}$ \\
Diabetes,\% & $1681(31.12)$ & $779(14.42)$ & $902(16.70)$ & 0.635 \\
Hypertension, \% & $3685(68.22)$ & $1688(31.25)$ & $1997(36.97)$ & $0.046^{*}$ \\
Smoke(years), 95\% Cl & $1275(23.60)$ & $1237(22.90)$ & $38(0.70)$ & $0.000^{*}$ \\
Overall max-IAD (mm) & $15.0 \pm 2.7$ & $15.6 \pm 2.8$ & $14.4 \pm 2.4$ & $0.001^{*}$ \\
\hline
\end{tabular}

a abdominal aortic aneurysm; ${ }^{b}$ coronary artery disease; ${ }^{\mathrm{C}}$ The maximum infrarenal aortic diameter; ${ }^{*} P<0.05$ 
$30 \%$ cohort were obese or overweight (BMI $\geqq 26 \mathrm{~kg} / \mathrm{m}^{2}$ ). Additionally, the mean Max-IAD of the screened population was $15.0 \pm 2.7 \mathrm{~mm}$ (range from 4.1 to $51.5 \mathrm{~mm}$, Figs. 1 and 2). The mean Max-IADs of different age groups were $14.9 \pm 2.3 \mathrm{~mm}$ (under 55 years), $15.1 \pm 2$. $7 \mathrm{~mm}$ (55-65 years), $15.0 \pm 3.0 \mathrm{~mm}$ (65-75 years) and $14.3 \pm 2.7 \mathrm{~mm}$ (over 75 years), respectively. Furthermore, the Max-IAD in females was smaller than in males $(14.4 \pm 2.4 \mathrm{~mm}$ vs $15.6 \pm 2.8 \mathrm{~mm}, P=0.001$, Table 1$)$. Both the mean and median Max-IAD of the total population were $15 \mathrm{~mm}$ (95\% CI, 14.9 to $15.0 \mathrm{~mm}$, Fig. 2).

\section{Epidemiological data and risk factors of AAA}

Eighteen AAAs were detected during the study, with total prevalence rate of $0.33 \%$ in the eligible screened individuals. Clinical characteristics of AAAs were shown in Tables 2 and 3. The prevalence rate of AAA in male was higher than in female $(0.55 \%$ vs $0.14 \%, P=0.009)$. The difference in mean age between men $(57.1 \pm 10$. 5 years) and women (71.3 \pm 11.2 years) was statistically significant $(P=0.001)$. Additionally, the prevalence rate of AAA had significant differences in different age groups, and results showed a higher AAA prevalence in age $55-75$ years than that in the other age groups $(0.51 \%$ vs $0.11 \%, P=0.016)$. The prevalence rate of AAA in the at-risk urban population was higher than that in the atrisk rural population $(0.50 \%$ vs $0.18 \%, P=0.041)$. Moreover, the diameter of aneurysms ranged from 28 to 51 . $5 \mathrm{~mm}$ (mean Max-IAD was $35.1 \pm 6.0 \mathrm{~mm}$ ). Above $80 \%$ of all screening-detected AAAs had over $40 \mathrm{~mm}$ in aneurysms diameter, and the size distribution of Max-IAD over $28 \mathrm{~mm}$ (Fig. 1).

The risk factor distribution was displayed in Tables 2 and 3. AAAs with the old-age, history of stroke, hypertension, CAD, TC, LDL, positive family history of AAA,

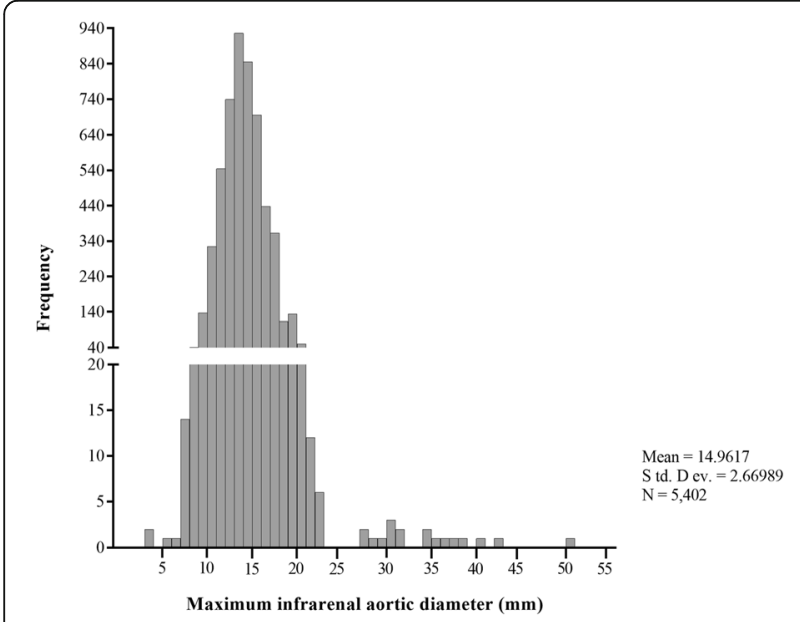

Fig. 1 The distribution of maximum infrarenal aortic diameters for the entire screened cohort (including a selective histogram of the size distribution of infrarenal aortic diameters $>28 \mathrm{~mm}$ ) smoking and drinking history had higher morbidity than AAAs without these risk factors $(P<0.05)$. Additionally, according to the results of multivariable regression analysis for predictors of AAA, history of stroke, CAD, smoking history, positive family history and $\mathrm{TC}$ value were independently associated with AAA (Table 4).

\section{Discussion}

AAA is a progressive disease with increasing age. The high prevalence of AAA in Caucasian population is well reported [10, 16]. Early detection of AAA can save life, and offer a much safe and effective treatment to emergency intervention of AAA rupture patients [2]. However, the screening target population is different according to the different recommendations [17-19]. The Society for Vascular Surgery recommended an AAA screening for women aged 65 years or older who have smoked or have a family history of AAA and men aged 55 years or older who have a family history of AAA [18]. Despite current studies recommend screening for high-risk groups, it has been also debated that who should be screened and which risk factors can potentially be used to confirm high-risk population for cost-effective of screening [20-22]. A previous study found that about half of the patients with AAA were not screened according to the current guidelines [8]. Moreover, it is also doubtful that the results of the target-population screening reported by Western studies are suitable for Chinese screening for AAA. Consequently, our present study expanded the screening target population. We screened subjects aged over 40 years who have well-known risk factors related to AAA, aiming to explore detailed information on epidemiological data of AAA in Chinese population.

There are few studies on accurate prevalence rate of AAA in Chinese population. It has been reported that the mean Max-IAD was about $15 \mathrm{~mm}$, and prevalence rate of AAA was $0.11 \%$ in the Chinese hypertensive adults, which showed smaller mean abdominal aortic size and lower prevalence of AAA in Chinese population than in Western population [23]. A previous study also showed that the mean Max-IAD in China was $17.5 \mathrm{~mm}$ for men and $14.8 \mathrm{~mm}$ for women [13]. Therefore, a diagnosis of AAA with Max-IAD of $>30 \mathrm{~mm}$ in China may be questioned. Our results showed small abdominal aortic size of the mean Max-IAD was $15.0 \pm 2.7 \mathrm{~mm}$ in the overall screened population. According to the recommendation of the Society of Vascular Surgery and International Society for Cardiovascular Surgery, we defined AAA as an Max-IAD of $>30 \mathrm{~mm}$ or having $50 \%$ increase or more in diameter compared with the mean normal [24]. We found that the prevalence rate of AAA was low and only 18 AAAs were found $(0.33 \%)$. The result was similar to the previous study [14, 23]. Based on our present study, there was a lower prevalence rate of AAA 

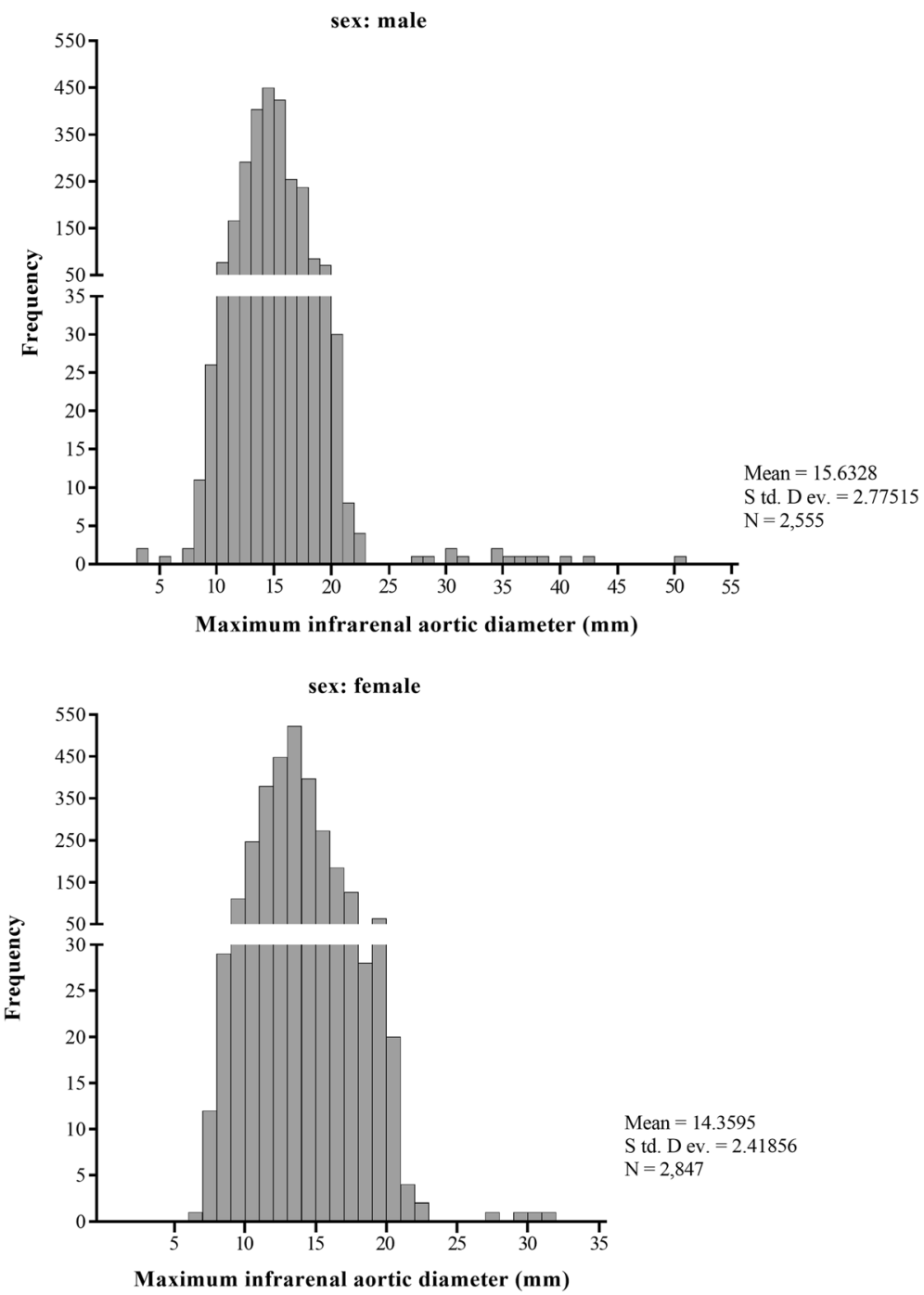

Fig. 2 The distribution of maximum infrarenal aortic diameters for the male and female at-risk individuals

when its definition as Max-IAD of $>30 \mathrm{~mm}$, only 18 AAA patients $(\mathrm{AD}>30 \mathrm{~mm})$ in our screened individuals.

Future screening strategies for AAA should be targeted those high-risk individuals. The United States Preventive Services Task Force (USPSTF) commanded that risk factors for AAA included older age, male sex, smoking history, positive family history of aneurysms, hypertension, cerebrovascular disease, coronary artery disease, atherosclerosis, hypercholesterolemia and obesity [17]. Our results showed that the prevalence rate of AAA was significantly higher in men than in women, and male was older ( $71.3 \pm 11.2$ years) than female $(57.1 \pm 10.5$ years $)$ in the total AAAs, which were consistent with previously reported results $[10,14]$. The screened individuals aged 55-75 years had a higher AAA prevalence rate than other age groups. Our result confirmed this observation that the at-risk male aged 55-75 years should be targeted for further screening because of the high prevalence rate of AAA.

The geographic areas of our screening program contained a significant difference between urban and rural areas with different dietary and lifestyle habits. The prevalence rate of AAA in the urban population was higher than in the rural population, which might be due to the higher consumption of fast food, meat and lack of exercise in urban population. This result reinforced the role of lifestyle in forming AAA [8]. Meanwhile, the result of our study reaffirmed that smoking, drinking history, positive family history of AAA, history of stroke, CAD, hypertension and hyperlipidemia were more likely to increase the prevalence of AAA. We found that history of stroke, CAD, smoking history, TC value and positive family history were independently associated with AAA. It has been proved that smoking as the major environmental risk 
Table 2 Characteristics of the screening at-risk cohort with and without abdominal aortic aneurysm (AAA) by age, gender, screening areas and medical history

\begin{tabular}{|c|c|c|c|}
\hline variables & $\begin{array}{l}\text { Without AAA } \\
(n=5384)\end{array}$ & $\begin{array}{l}\text { With AAA } \\
(n=18)\end{array}$ & $P$ \\
\hline \multicolumn{4}{|l|}{ Sex } \\
\hline Male, $\%$ & $2541(47.20)$ & $14(77.78)$ & $0.009^{*}$ \\
\hline Female,\% & $2843(52.80)$ & $4(22.22)$ & $0.009^{*}$ \\
\hline \multicolumn{4}{|l|}{ Age group } \\
\hline$\leq 55$ yrs.,\% & $1682(31.24)$ & 0 & \\
\hline$>55, \leq 65$ yrs., $\%$ & $1940(36.03)$ & $8(44.44)$ & $0.016^{*}$ \\
\hline$>65, \leq 75$ yrs., $\%$ & $1210(22.47)$ & $8(44.44)$ & $0.016^{*}$ \\
\hline$>75$ yrs & $552(10.25)$ & $2(11.11)$ & $0.016^{*}$ \\
\hline \multicolumn{4}{|l|}{ Screening areas } \\
\hline Urban,\% & $2589(48.09)$ & $13(72.22)$ & $0.041^{*}$ \\
\hline Rural,\% & 2795 (51.91) & $5(27.78)$ & $0.041^{*}$ \\
\hline \multicolumn{4}{|l|}{ Medical history } \\
\hline$C A D, \%^{a}$ & $975(18.11)$ & $8(44.44)$ & $0.004^{*}$ \\
\hline Diabetes, $\%$ & $1677(31.15)$ & $4(22.22)$ & 0.061 \\
\hline Hypertension,\% & $3668(68.13)$ & $17(94.44)$ & $0.001^{*}$ \\
\hline History of stroke,\% & $1470(27.30)$ & $11(61.11)$ & $0.001^{*}$ \\
\hline Family history of $\mathrm{AAA}, \%^{\mathrm{b}}$ & $2(0.04)$ & $3(16.67)$ & $0.000^{*}$ \\
\hline
\end{tabular}

${ }^{a}$ coronary artery disease; ${ }^{b}$ abdominal aortic aneurysm; ${ }^{*} P<0.05$

Table 3 Characteristics of the screening at-risk cohort with and without abdominal aortic aneurysm (AAA) by risk factors

\begin{tabular}{|c|c|c|c|}
\hline Risk factor & $\begin{array}{l}\text { Without AAA } \\
(n=5384) 95 \% \mathrm{Cl}\end{array}$ & $\begin{array}{l}\text { With AAA }(n=18) \\
95 \% \mathrm{Cl}\end{array}$ & $P$ \\
\hline Mean age & $61.15(60.87-61.43)$ & $68.00(62.81-73.19)$ & $0.005^{*}$ \\
\hline $\begin{array}{l}\text { Hypertension } \\
\text { (years) }\end{array}$ & $5.55(5.34-5.77)$ & $7.22(4.13-10.31)$ & 0.379 \\
\hline Smoke (years) & $6.43(6.08-6.78)$ & $21.28(11.09-31.47)$ & $0.000^{*}$ \\
\hline $\begin{array}{l}\text { Drinking history } \\
\text { (years) }\end{array}$ & $5.62(5.30-5.94)$ & $16.39(5.57-22.20)$ & $0.000^{*}$ \\
\hline $\mathrm{BMI}\left(\mathrm{kg} / \mathrm{m}^{2}\right)$ & 32.11 (24.29-39.93) & $25.58(23.51-27.65)$ & 0.925 \\
\hline $\operatorname{SBP}(m m H g)^{a}$ & $141.46(140.95-141.98)$ & $142.89(134.81-150.97)$ & 0.753 \\
\hline $\mathrm{DBP}(\mathrm{mmHg})^{\mathrm{b}}$ & $88.93(88.63-89.23)$ & $87.94(83.78-92.11)$ & 0.713 \\
\hline $\mathrm{FBG}(\mathrm{mmol} / \mathrm{L})^{\mathrm{c}}$ & $6.65(6.59-6.70)$ & $6.79(5.61-7.97)$ & 0.770 \\
\hline $\begin{array}{l}\text { Triglyceride } \\
(\mathrm{mmol} / \mathrm{L}) \mid\end{array}$ & $2.26(2.20-2.33)$ & $1.99(0.94-3.03)$ & 0.630 \\
\hline $\mathrm{TC}(\mathrm{mmol} / \mathrm{L})^{d}$ & $5.18(5.13-5.23)$ & $6.58(2.33-10.82)$ & $0.003^{*}$ \\
\hline $\mathrm{LDL}(\mathrm{mmol} / \mathrm{L})^{\mathrm{e}}$ & $2.91(2.89-2.93)$ & $2.47(2.15-2.78)$ & $0.028^{*}$ \\
\hline $\mathrm{HDL}(\mathrm{mmol} / \mathrm{L})^{f}$ & $1.40(1.38-1.42)$ & $1.42(1.19-1.65)$ & 0.929 \\
\hline $\begin{array}{l}\text { Homocysteine } \\
(\mu \mathrm{mol} / \mathrm{L})\end{array}$ & 18.96 (18.58-19.33) & 19.89 (16.00-23.77) & 0.780 \\
\hline
\end{tabular}

${ }^{\mathrm{a}}$ systolic blood pressure; ${ }^{\mathrm{b}}$ diastolic blood pressure; ${ }^{\mathrm{c}}$ fasting blood-glucose; ${ }^{d}$ serum total cholesterol; ${ }^{e}$ low density lipoproteins; ${ }^{\text {f }}$ high density lipoprotein; gabdominal aortic aneurysm; * $P<0.05$
Table 4 Results of multivariable regression analysis for predictors of abdominal aortic aneurysm (AAA)

\begin{tabular}{|c|c|c|}
\hline Risk factor & Odds ratio & $95 \% \mathrm{Cl}$ \\
\hline History of stroke & 0.244 & $0.088-0.677$ \\
\hline$C A D^{a}$ & 0.263 & $0.095-0.728$ \\
\hline Diabetes & 1.182 & $0.787-1.774$ \\
\hline Family history of $A A A^{b}$ & 1.3 & $1.1-1.4$ \\
\hline Hypertension & 1.006 & $0.953-1.062$ \\
\hline Smoking & 1.049 & $1.018-1.081$ \\
\hline Drinking history & 1.017 & $0.985-1.049$ \\
\hline BMI & 0.934 & $0.814-1.072$ \\
\hline $\mathrm{SBP}^{\mathrm{C}}$ & 1.006 & $0.977-1.035$ \\
\hline $\mathrm{DBP}^{\mathrm{d}}$ & 1.010 & $0.961-1.062$ \\
\hline $\mathrm{FBG}^{\mathrm{e}}$ & 1.101 & $0.898-1.350$ \\
\hline Triglyceride & 0.969 & $0.745-1.262$ \\
\hline$T C^{f}$ & 1.092 & $1.025-1.164$ \\
\hline $\operatorname{LDL}^{\mathrm{g}}$ & 0.493 & $0.251-0.968$ \\
\hline$H D L^{h}$ & 1.082 & $0.592-1.977$ \\
\hline Homocysteine & 1.000 & $0.967-1.034$ \\
\hline
\end{tabular}

${ }^{a}$ coronary artery disease; ${ }^{b}$ abdominal aortic aneurysm; ${ }^{c}$ systolic blood pressure; ${ }^{\mathrm{d}}$ diastolic blood pressure; ${ }^{\mathrm{e}}$ fasting blood-glucose; ${ }^{\mathrm{f}}$ serum total cholesterol; ' Iow density lipoproteins; ${ }^{\text {h}}$ high density lipoprotein

factor has a strong positive association with AAA, which can increase the prevalence and the risk of rupture of AAA [8]. Many patients with AAA often have other atherosclerotic vascular diseases, which is the same as our patients, and these diseases have common risk factors [8]. It is also known that hyperlipidemia is involved in the formation of vascular wall atherosclerosis and AAA. Atherosclerotic vascular diseases and AAA have been traditionally regarded as two separate angiosis but have some common pathogenetic backgrounds [1]. Previous studies indicated that high level of homocysteine played a role in the pathogenesis of atherosclerotic vascular disease [25]. The present study found that plasma homocysteine level was not directly associated with AAA, which indicated that atherosclerosis and AAA had different pathogenesis.

However, this study had several limitations. Our study was composed of a highly selected group of subjects with one or several risk factors of arteriosclerotic diseases. The present screening trial was mainly only focused on the residents of several local communities, and only 18 AAAs were found due to the low prevalence of AAA in Middle China. Meanwhile, because of lacking long-term follow-up, our study was a cross-sectional study, and the long-term benefit of screening was uncertain. Therefore, the large-scale screening study is indispensable to validate our finding. 


\section{Conclusions}

This was the first report on the large screening program for AAA in residents of the urban and rural communities in Middle China. This study provided a good evidence that the mean Max-IAD in Chinese population was apparently small by comparison with other reports. The result of significantly low prevalence rate of AAA in highly selected at-risk group did not support routine screening in Chinese population. The male aged 5575 years should be targeted for further screening because of the high prevalence rate of AAA. The results of our study also confirmed that smoking, drinking history, atherosclerotic burden, hypertension, hyperlipidemia and positive family history of AAA were more likely to increase the prevalence rate of AAA.

\begin{abstract}
Abbreviations
AAA: Abdominal aortic aneurysm; BMI: Body mass index; CAD: Coronary artery disease; HDL: High density lipoprotein; LDL: Low density lipoproteins; Max-IAD: Maximum infrarenal aortic diameter; TC: Total cholesterol; USPSTF: The United States Preventive Services Task Force
\end{abstract}

\section{Acknowledgements}

We gratefully acknowledge the National Science Foundation of China and Henan provincial Medical Science and technology research program.

\section{Ethical approval and consent to participate}

The study was approved by the Ethics Committee of Zhengzhou University (HSY2014047). All screened individuals consented to the use of their anonymity clinical data of all screening program for the purpose of research.

\section{Funding}

The study was supported by the National Natural Science Foundation of China (grant number 81301328,2013 ) and Henan provincial Medical Science and technology research program (grant number 201301011, 2013).

\section{Availability of data and materials}

The datasets used and/or analysed during the current study are available from the corresponding author on reasonable request.

\section{Authors' contributions}

L.K. and Z.S. conceived and designed the experiments and wrote the manuscript Z.K. performed the experiments and analyzed the data. L.T. contributed to the quality control of data and algorithms. All authors read and approved the final manuscript.

\section{Consent for publication}

Not applicable.

\section{Competing interests}

The authors declare that they have no competing interests.

\section{Publisher's Note}

Springer Nature remains neutral with regard to jurisdictional claims in published maps and institutional affiliations.

Received: 28 April 2017 Accepted: 20 March 2018

Published online: 03 April 2018

\section{References}

1. Cornuz J, Pinto CS, Tevaearai H, et al. Risk factors for asymptomatic abdominal aortic aneurysm. Eur J Pub Health. 2004;14(4):343-9.

2. Chun KC, Teng KY, Van Spyk EN, et al. Outcomes of an abdominal aortic aneurysm screening program. J Vasc Surg. 2013;57(2):376-81.
3. Guirguis-Blake JM, Beil TL, Senger CA, et al. Ultrasonography screening for abdominal aortic aneurysms: a systematic evidence review for the U.S. preventive services task force. Ann Intern Med. 2014;160(5):321-9.

4. Mussa FF. Screening for abdominal aortic aneurysm. J Vasc Surg. 2015;62(3):774-8

5. Thompson SG, Ashton HA, Gao L, et al. Final follow-up of the multicentre aneurysm screening study (MASS) randomized trial of abdominal aortic aneurysm screening. Br J Surg. 2012;99:1649-56.

6. Lindholt JS, Juul $\mathrm{S}$, Fasting $\mathrm{H}$, et al. Preliminary ten year results from a randomised single Centre mass screening trial for abdominal aortic aneurysm. Eur J Vasc Endovasc Surg. 2006;32(6):608-14.

7. Kuivaniemi H, Elmore JR. Opportunities in abdominal aortic aneurysm research: epidemiology, genetics, and pathophysiology. Ann Vasc Surg. 2012;26(6):862-70

8. Kent KC, Zwolak RM, Egorova NN, et al. Analysis of risk factors for abdomina aortic aneurysm in a cohort of more than 3 million individuals. J Vasc Surg. 2010;52(3):539-48.

9. Cueff $C$, Keenan NG, Krapf $L$, et al. Screening for abdominal aortic aneurysm in coronary care unit patients with acute myocardial infarction using portable transthoracic echocardiography. Eur Heart J Cardiovasc Imaging. 2012;13(7):574-8.

10. Yii MK. Epidemiology of abdominal aortic aneurysm in an Asian population. ANZ J Surg. 2003;73(6):393-5

11. Poon JT, Cheng SW, Wong JS, et al. Prevalence of abdominal aortic aneurysm in Chinese patients with severe coronary artery disease. ANZ J Surg. 2010;80(9):630-3

12. Adachi K, Iwasawa T, Ono T. Screening for abdominal aortic aneurysms during a basic medical checkup in residents of a Japanese rural community. Surg Today. 2000;30(7):594-9.

13. Salem MK, Rayt HS, Hussey G, et al. Should Asian men be included in abdominal aortic aneurysm screening programmes? Eur J Vasc Endovasc Surg. 2009;38(6):748-9

14. Benson RA, Poole R, Murray $\mathrm{S}$, et al. Screening results from a large United Kingdom abdominal aortic aneurysm screening center in the context of optimizing United Kingdom National Abdominal Aortic Aneurysm Screening Programme protocols. J Vasc Surg. 2015;63(2):301-4.

15. Wanhainen A, Björck $M$. The Swedish experience of screening for abdominal aortic aneurysm. J Vasc Surg. 2011;53(4):1164-5.

16. Hafez $\mathrm{H}$, Druce PS, Ashton HA. Abdominal aortic aneurysm development in men following a "normal" aortic ultrasound scan. Eur J Vasc Endovasc Surg. 2008;36(5):553-8.

17. Lefevre ML. Screening for abdominal aortic aneurysm: U.S. preventive services task force recommendation statement. Ann Intern Med. 2014;161(4):281-90.

18. Chaikof EL, Brewster DC, Dalman RL, et al. SVS practice guidelines for the care of patients with an abdominal aortic aneurysm: executive summary. J Vasc Surg. 2009;50(4):880-96.

19. Moll FL, Powell JT, Fraedrich G, et al. Management of abdominal aortic aneurysms clinical practice guidelines of the European society for vascular surgery. Eur J Vasc Endovasc Surg. 2011;41(Suppl 1):S1-S58.

20. Wanhainen A, Svensjö S, Mani K. Screening for abdominal aortic aneurysmareas where information is still inadequate. Scand J Surg. 2008;97(2):131-5.

21. Mureebe L, Egorova N, Giacovelli JK, et al. National trends in the repair of ruptured abdominal aortic aneurysms. J Vasc Surg. 2008;48(5):1101-7.

22. Kung HC, Hoyert DL, Xu J, et al. Deaths: final data for 2005. Natl Vital Stat Rep. 2008;56(10):1-120.

23. Wei R, Liu LS, Wang LW, et al. Association of Resting Heart Rate with Infrarenal aortic diameter: a cross sectional study in Chinese hypertensive adults. Eur J Vasc Endovasc Surg. 2015;50(6):714-21.

24. Johnston KW, Rutherford RB, Tilson MD, et al. Suggested standards for reporting on arterial aneurysms. J Vasc Surg. 1991;13(3):452-8.

25. Moroz P, Le MT, Norman PE. Homocysteine and abdominal aortic aneurysms. ANZ J Surg. 2007;77(5):329-32. 